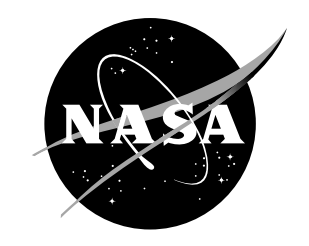

\title{
The Effect of Boundary Support and Reflector Dimensions on Inflatable Parabolic Antenna Performance
}

Michael J. Coleman and Frank Baginski

George Washington University, Washington, DC

Robert R. Romanofsky

Glenn Research Center, Cleveland, Ohio 


\section{NASA STI Program . . . in Profile}

Since its founding, NASA has been dedicated to the advancement of aeronautics and space science. The NASA Scientific and Technical Information (STI) program plays a key part in helping NASA maintain this important role.

The NASA STI Program operates under the auspices of the Agency Chief Information Officer. It collects, organizes, provides for archiving, and disseminates NASA's STI. The NASA STI program provides access to the NASA Aeronautics and Space Database and its public interface, the NASA Technical Reports Server, thus providing one of the largest collections of aeronautical and space science STI in the world. Results are published in both non-NASA channels and by NASA in the NASA STI Report Series, which includes the following report types:

- TECHNICAL PUBLICATION. Reports of completed research or a major significant phase of research that present the results of NASA programs and include extensive data or theoretical analysis. Includes compilations of significant scientific and technical data and information deemed to be of continuing reference value. NASA counterpart of peer-reviewed formal professional papers but has less stringent limitations on manuscript length and extent of graphic presentations.

- TECHNICAL MEMORANDUM. Scientific and technical findings that are preliminary or of specialized interest, e.g., quick release reports, working papers, and bibliographies that contain minimal annotation. Does not contain extensive analysis.

- CONTRACTOR REPORT. Scientific and technical findings by NASA-sponsored contractors and grantees.
- CONFERENCE PUBLICATION. Collected papers from scientific and technical conferences, symposia, seminars, or other meetings sponsored or cosponsored by NASA.

- SPECIAL PUBLICATION. Scientific, technical, or historical information from NASA programs, projects, and missions, often concerned with subjects having substantial public interest.

- TECHNICAL TRANSLATION. Englishlanguage translations of foreign scientific and technical material pertinent to NASA's mission.

Specialized services also include creating custom thesauri, building customized databases, organizing and publishing research results.

For more information about the NASA STI program, see the following:

- Access the NASA STI program home page at http://www.sti.nasa.gov

- E-mail your question via the Internet to help@ sti.nasa.gov

- Fax your question to the NASA STI Help Desk at $443-757-5803$

- Telephone the NASA STI Help Desk at 443-757-5802

- Write to: NASA Center for AeroSpace Information (CASI) 7115 Standard Drive Hanover, MD 21076-1320 
NASA/TM—2011-217110

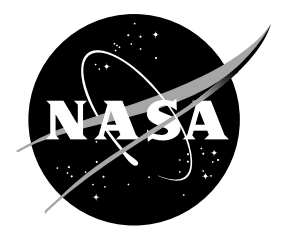

\section{The Effect of Boundary Support and Reflector Dimensions on Inflatable Parabolic Antenna Performance}

Michael J. Coleman and Frank Baginski

George Washington University, Washington, DC

Robert R. Romanofsky

Glenn Research Center, Cleveland, Ohio

National Aeronautics and

Space Administration

Glenn Research Center

Cleveland, Ohio 44135 


\section{Acknowledgments}

The first two authors were supported by NASA Grants NNX07AR67G and NNX09AH08G.

Trade names and trademarks are used in this report for identification only. Their usage does not constitute an official endorsement, either expressed or implied, by the National Aeronautics and Space Administration.

Level of Review: This material has been technically reviewed by technical management.

Available from

NASA Center for Aerospace Information 7115 Standard Drive

Hanover, MD 21076-1320
National Technical Information Service 5301 Shawnee Road Alexandria, VA 22312

Available electronically at http://www.sti.nasa.gov 


\title{
The Effect of Boundary Support and Reflector Dimensions on Inflatable Parabolic Antenna Performance
}

\author{
Michael J. Coleman and Frank Baginski \\ George Washington University \\ Washington, DC 20052 \\ Robert R. Romanofsky \\ National Aeronautics and Space Administration \\ Glenn Research Center \\ Cleveland, Ohio 44135
}

\begin{abstract}
For parabolic antennas with sufficient surface accuracy, more power can be radiated with a larger aperture size. This paper explores the performance of antennas of various size and reflector depth. The particular focus is on a large inflatable elastic antenna reflector that is supported about its perimeter by a set of elastic tendons and is subjected to a constant hydrostatic pressure. The surface accuracy of the antenna is measured by an RMS calculation, while the reflector phase error component of the efficiency is determined by computing the power density at boresight. In the analysis, the calculation of antenna efficiency is not based on the Ruze Equation. Hence, no assumption regarding the distribution of the reflector surface distortions is presumed. The reflector surface is modeled as an isotropic elastic membrane using a linear stress-strain constitutive relation. Three types of antenna reflector construction are considered: one molded to an ideal parabolic form and two different flat panel design patterns. The flat panel surfaces are constructed by seaming together panels in a manner that the desired parabolic shape is approximately attained after pressurization. Numerical solutions of the model problem are calculated under a variety of conditions in order to estimate the accuracy and efficiency of these antenna systems. In the case of the flat panel constructions, several different cutting patterns are analyzed in order to determine an optimal cutting strategy.
\end{abstract}




\section{Nomenclature}

A Aperture area of the antenna (square meters)

$D \quad$ Diameter of antenna aperture (meters)

$E_{f} \quad$ Gravitational potential energy due to film (Joules)

$E_{p} \quad$ Hydrostatic pressure potential energy (Joules)

$E_{T} \quad$ Total energy of the inflatable reflector system (Joules)

$F \quad$ Focal length of antenna (meters)

$G \quad$ Effective antenna gain

$G_{I} \quad$ Ideal antenna gain

$g \quad$ Acceleration due to gravity (meters per square second)

$K_{t, m}$ Tendon stiffness constant in the $m^{\text {th }}$ supporting tendon (Newtons)

$S_{f}^{*} \quad$ Relaxed film strain energy (Joules)

$S_{t}^{*} \quad$ Relaxed tendon strain energy (Joules)

$N \quad$ Number of triangular elements in the upper and lower shells

$N_{f} \quad$ Number of triangular elements in the lower shell

$N_{t} \quad$ Number of supporting tendons

$p_{0} \quad$ Constant differential pressure (Pascals)

$W_{f}^{*} \quad$ Relaxed film strain energy density (Joules per square meter)

$W_{t, m}^{*}$ Relaxed tendon strain energy density in the $m^{\text {th }}$ supporting tendon (Joules per square meter)

$E \quad$ Young's modulus of film (Pascals)

$\nu \quad$ Poisson's ratio of film material

$\Omega_{R} \quad$ Reference configuration

$\Omega \quad$ Deformed configuration

$\alpha \quad$ Rim modification factor

$\gamma \quad$ Tendon length adjustment factor

$\varepsilon_{m} \quad$ Strain in the $m^{\text {th }}$ supporting tendon (meters per meter)

$\lambda \quad$ Radiation wavelength (meters)

$f \quad$ Radiation frequency (Hertz)

$\mathbb{R}^{k} \quad k$-dimensional Euclidean space

$\hat{\mathbf{r}} \quad$ Unit direction vector from the antenna to a point in the far field (unit meter)

$\hat{\mathbf{j}} \quad$ Unit direction vector $(0,1,0)$ (unit meter)

$\hat{\mathbf{k}} \quad$ Unit direction vector $(0,0,1)$ (unit meter) 


\section{Introduction}

The future of communication in near Earth orbit and deep space missions may require large deployable circular aperture antennas. It is conceivable that the diameter of the aperture may need to reach 10 meters or more in order to support data transmission rates of hundreds of megabits per second. Similarly, much larger apertures may be required for futuristic remote sensing and imaging applications. ${ }^{1}$ A rigid reflector of such large diameter, however, will not fit aboard a small launch vehicle for transport into orbit. Deployable space structures are advantageous in deep-space and near Earth orbit missions due to their low construction expense, low weight and high packing efficiency. ${ }^{2}$ For these reasons, the use of deployable antenna reflectors is an attractive option.

Although inflatable antennas have been considered since the 1960s with successful deployment for L and $\mathrm{S}$ band applications, ${ }^{3}$ there is now interest in using such antennas for shorter wavelength bands. There are three fundamental deployable antenna families, each with its own set of attributes: mesh reflectors with various deployment mechanisms, shape memory polymer and tensioned membrane shells, and the inflatable membrane reflectors under consideration here. Mesh reflectors dominate the space arena and have an impressive 20 year plus track record. Nevertheless, there have been a number of deployment anomalies compromising mission success, including NASA's Galileo satellite, the Asia Cellular Satellite, the Japanese VLBI Space Observatory Program, and most recently SkyTerra 1. Shape memory polymer reflectors are a nascent concept and perhaps at too low a technology readiness level to predict future mission acceptance. These observations make the case for an alternative antenna technology, namely inflatable membranes, which promise less complex deployment mechanisms (hence higher reliability), substantially lower aerial density, and superior packaging efficiency.

It is critical that the reflector have minimal surface distortions and retain a parabolic shape, particularly when operating in frequencies with shorter wavelengths (Ka-Band, for example). One estimate for the tolerance of the reflector surface accuracy is an RMS value less than 0.5 millimeters. ${ }^{1}$ A common method for calculating antenna efficiency directly from RMS involves the Ruze Equation. While the Ruze Equation simplifies the computation of antenna efficiency, applying it requires the assumption of random reflector surface distortions having a Gaussian distribution. ${ }^{4}$ Some earlier research has questioned the accuracy of the Ruze Equation in analyzing inflatable antenna efficiency. This is specifically a concern since the surface errors tend to concentrate near the antenna rim. ${ }^{1}$ 
The purpose of this paper is to investigate the elastic deformation of inflatable antennas in order to more carefully analyze the areas of deformation, material stresses, regions of potential wrinkling and the effect of these characteristics on the overall antenna efficiency. In the model presented, the reflector of an inflatable antenna is treated as an elastic membrane which deforms in response to external forces. The model employs a finite element method to directly compute the power gain of a given deformed reflector. A key advantage of this analysis is that the Ruze Equation is not used and therefore no assumption is made regarding the distribution of reflector surface distortions.

Earlier work by Greschik ${ }^{5}$ showed that the precision of the membrane shape is sensitive to membrane properties and wrinkling. Previous work by the authors, explored non-symmetric boundary and gravitational loading and found that asymmetric boundary conditions or high supporting tendon forces can cause substantial distortion to the reflector surface. ${ }^{6}$ Reflector construction methods (cutting patterns) were also explored to help reduce the surface error. Some preliminary findings suggested that modifications to the cutting patterns could help reduce the deflection of the reflector's rim and thereby improve the surface accuracy. ${ }^{7}$

This paper extends some earlier results ${ }^{8}$ for the flat panel constructions of small antennas to larger antenna sizes. In addition, two parametric studies focusing on both size and depth of the antenna reflector are presented. The shape of the deformed membrane is calculated using a model for pneumatic envelopes developed by the second author and his collaborators..$^{9,10}$ The geometry of the discretized model for a molded reflector is presented in Section III while the geometry of the flat panel constructions are described in Section IV. Section V introduces RMS calculations that are used to evaluate the antenna shapes obtained. These calculations are based on a numerical model that approximates the reflector as a faceted surface. No assumption about the distribution of surface errors is made, nor is the Ruze Equation applied in computation of the antenna efficiency. Finally, some numerical results will be presented in Section VI. The results include parametric studies that vary the tendon forces, size of the aperture and depth of the antenna reflector.

\section{Basic Antenna Design}

The inflatable antenna system considered in this study is formed by molding a light-weight material into parabolic form. Two such parabolic membranes are created and seamed along their rims. The two mem- 
branes are held to parabolic shape by an inflation gas between them and are supported by tendons which are attached to the seamed rim of the membranes on one end and a rigid frame on the other. Typically, one of the parabolic sheets is coated with $1200 \AA$ of aluminum to make the surface a reflective one. The coated half of the closed surface will serve as the antenna's reflector. See Figure 1 for a schematic of the inflatable antenna system.

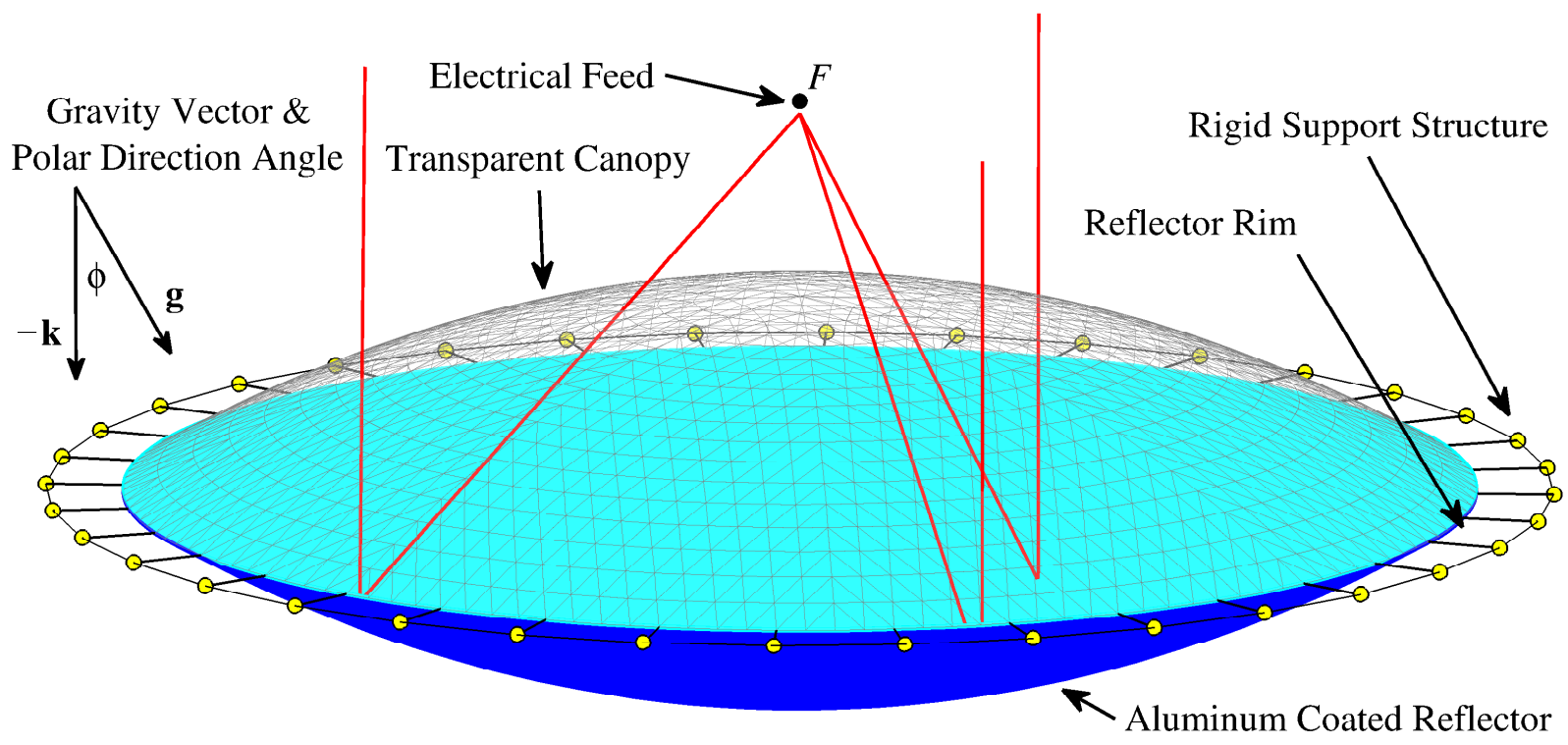

Figure 1. Parabolic reflector. The lines emanating from $F$ are typical light paths.

For large reflectors (diameters closer to the 10 meter range), the molding process may be infeasible or too expensive. One may therefore wish to consider models for a reflector constructed from flat panels of material that are cut and seamed in a manner to approximate the parabolic reflector surface. The ability of such a reflector to achieve the desired parabolic shape will be investigated. For these constructions, the accuracy of the pressurized reflector will depend on the cutting pattern.

\section{Faceted Surface Construction and Mathematical Model}

This section presents a mathematical model in which the membrane is approximated by a faceted surface, using constant-strain, plane-stress triangular finite elements. Figure 1 is an image of the actual faceted surface used for a molded reflector in computations. Although the coated reflector is labeled in Figure 1 for exposition, the effect of the vaporized aluminum is included on neither the reflective properties nor mechanical properties of the reflector. For these studies, it is assumed that the entire membrane is made of $12.7 \mu \mathrm{m}$ Kapton. The material parameters will therefore be identical for both the upper and lower paraboloids in these investigations. The mechanical properties of Kapton are presented in Table 1. 
In order to generate the faceted surface, a parabolic mold is created by the parametric equations

$$
x=a+r \cos \theta ; \quad y=a+r \sin \theta ; \quad z=r^{2} /(4 F),
$$

for $0 \leq \theta \leq 2 \pi$ and $0 \leq r \leq R_{f}$ where $R_{f}$ is the radius of the reflector aperture and $F$ is the focal length of the antenna. In Equations (1), $a \geq 0$ is a translation that is used to generate a mold for an off-axis reflector. For axisymmetric reflectors, set $a=0$. The paraboloid of Equations (1) is then translated and rotated so that the rim lies in the plane $z=0$ and the vertex lies on the $z$-axis. A symmetric paraboloid is formed over the plane $z=0$ to generate the canopy and produce a clam shell mold. The triangles of the faceted surface are formed by connecting vertices that initially lie on this mold. To simulate a molded reflector, one takes this initial configuration as the unstrained reference configuration $\Omega_{R} \subset \mathbb{R}^{3}$. Simulating the two flat panel constructions will involve a different unstrained reference configuration $\Omega_{R} \subset \mathbb{R}^{2}$ (see Section IV).

One of the parametric studies varies the depth of the parabolic reflector. The depth of the reflector is entirely determined by the diameter of the aperture, $D=2 R_{f}$ and the focal length of the antenna, $F$. The ratio $F / D$ is used to express the depth of the antenna. If the reflector size is fixed at a certain aperture diameter, then the depth is controlled by adjusting $F$. See Figure 2 for several antenna profiles having $D=2.13$ meters.

The supporting tendons are modeled as linearly elastic strings. When the envelope is pressurized, the entire inflatable reflector system (including the supporting tendons) will seek an equilibrium configuration of minimum potential energy. In the model, the pressure, tendon stiffness and gravity are treated as parameters which can be modified for parametric studies. Evaluating the shape for various pressure levels and orientations is of interest as the reflector is likely to operate in different gravitational environments.

This model follows an optimization-based solution process which determines the equilibrium position of the inflated reflector by minimizing the total energy of the discretized system. ${ }^{9}$ Consider a deformation $\mathbf{x}$ of the reference configuration $\Omega_{R} \subset \mathbb{R}^{k}$ for $k=2,3$. The deformed configuration is a surface in $\mathbb{R}^{3}$, i.e., $\mathbf{x}\left(\Omega_{R}\right)=\Omega \subset \mathbb{R}^{3}$. For a particular deformed configuration, the energy of the antenna system is calculated by the functional

$$
E_{T}(\mathbf{x})=E_{f}(\mathbf{x})+E_{p}(\mathbf{x})+S_{f}^{*}(\nabla \mathbf{x})+S_{t}^{*}(\nabla \mathbf{x})
$$

where $E_{f}$ is the gravitational potential energy of the film, $E_{p}$ is the hydrostatic pressure potential energy, 


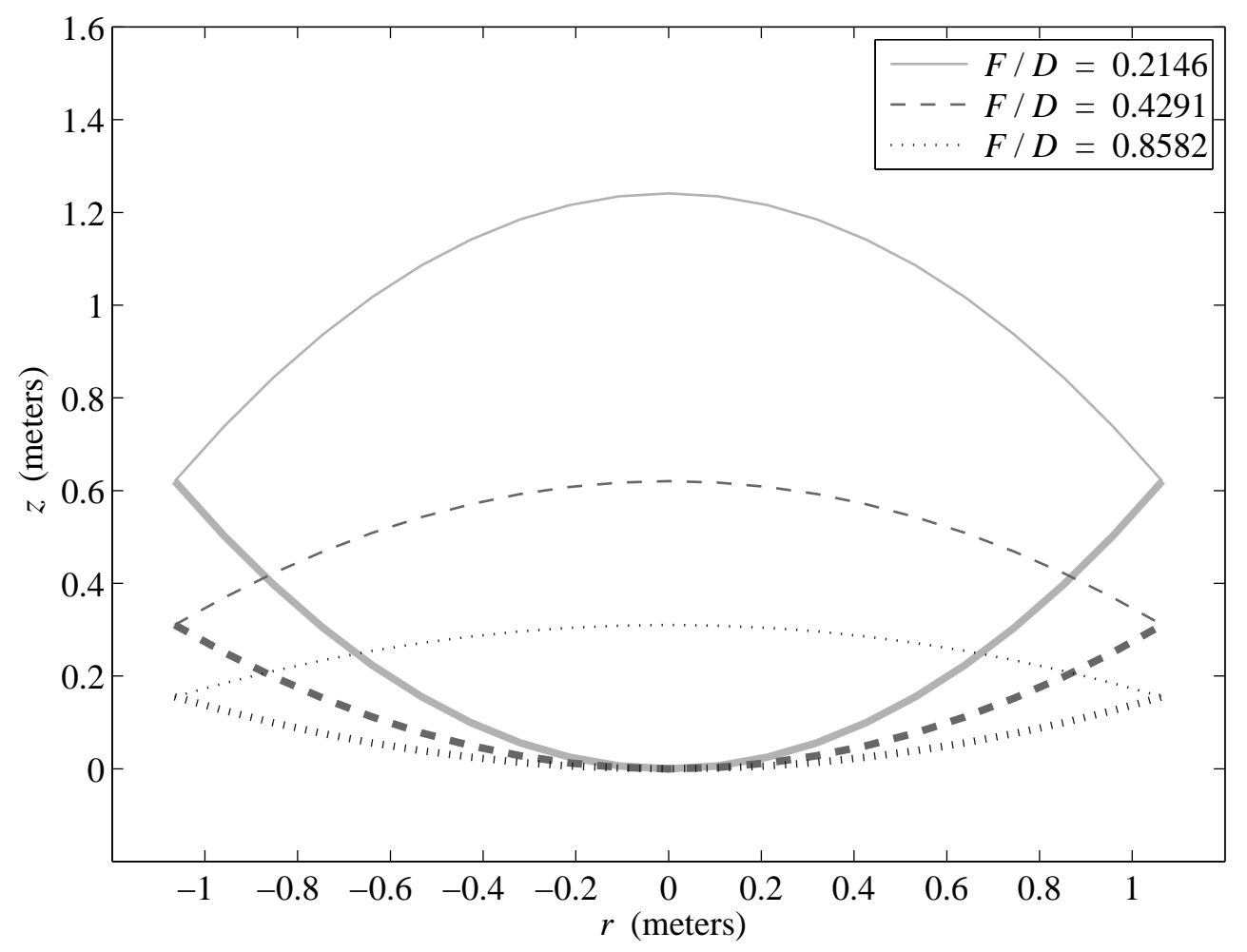

Figure 2. Cross section of inflated antennas for different $F / D$ values and $z=r^{2} /(4 F)$.

$S_{f}^{*}$ is the relaxed strain energy in the membrane and $S_{t}^{*}$ is the relaxed strain energy in the supporting elastic tendons. ${ }^{9}$

\section{III.A. Gravitational Potential}

The direction and magnitude of gravity are included as parameters in the model since the antennas in question may be operating in a variety of extraterrestrial environments. Given that these antennas are tested on the ground and used in space applications, one may wish to explore the effect of zero gravity, a $1 \mathrm{~g}$ environment and the gravitational environments of other planets. The total gravitational potential energy can be calculated by

$$
E_{f}(\mathbf{x})=\iint_{\Omega_{R}} w_{f}(\mathbf{x} \cdot \mathbf{g}) d A
$$

where $w_{f}$ is the film weight density, $\mathbf{g}$ is the directed acceleration due to gravity and $d A$ is the surface area measure over the faceted reference configuration $\Omega_{R}$. This quantity is calculated exactly for each triangular facet and then summed over the set of triangles.

For the remainder of this paper, fix the parameter $g=9.80665$ meters $/ \mathrm{sec}^{2}$ for the acceleration due to 
gravity. The only case for which the gravity vector $\mathbf{g}$ will not have the aforementioned magnitude is for a zero gravity environment.

\section{III.B. Hydrostatic Pressure Potential}

For the deformed configuration $\Omega$, let $B$ be the enclosed interior space. Assuming that the differential pressure is a constant $p_{0}$, the hydrostatic pressure potential energy is given by

$$
E_{p}(\mathbf{x})=\iiint_{B} p_{0} d V=\iint_{\Omega} p_{0} \mathbf{k} \cdot \hat{\mathbf{n}} d S
$$

where $\hat{\mathbf{n}}$ is the unit outward normal to the deformed surface $\Omega$ and $d S$ is surface area measure on $\Omega$. The divergence theorem was applied to obtain the last integral in Equation (3). The quantity $E_{p}$ is calculated exactly for the faceted surface $\Omega$.

\section{III.C. Film Strain Energy}

The strain energy in the film is calculated by

$$
S_{f}^{*}(\nabla \mathbf{x})=\iint_{\Omega_{R}} W_{f}^{*}(\nabla \mathbf{x}) d A
$$

where $d A$ is area measure in $\Omega_{R}$ and $W_{f}^{*}$ is the relaxation of $W_{f}$ (i.e., $W_{f}^{*}$ is the largest convex function that does not exceed $\left.W_{f}\right)$. The strain energy density is

$$
W_{f}=\frac{1}{2}(\mathbf{S}: \mathbf{G})
$$

where $\mathbf{G}$ is the Cauchy-Green strain tensor, $\mathbf{S}$ is the second Piola-Kirchoff stress resultant tensor, and ':' denotes the tensor inner product. A piecewise constant strain model is applied to the faceted surface. Each triangle has two principal stresses and two principal strains. The principal strains are the eigenvalues of $\mathbf{G}$ and are denoted $\delta_{1}$ and $\delta_{2}$. The principal stress resultants are the eigenvalues of $\mathbf{S}$ and are given by $\mu_{1}$ and $\mu_{2}$. A thin compliant membrane will not resist compressive stresses but wrinkle instead. The wrinkling is modeled by using the relaxed strain energy density, $W_{f}^{*}$. Following the method due to Pipkin, ${ }^{11}$ the faceted surface is partitioned into three sets: the set of slack facets, the set of wrinkled facets, and the set of taut facets. For any given triangular facet, $T \in \Omega_{R}$, the state is determined by the principal strains and stresses. The conditions of Equation (4) describe the possible states: (a) slack, (b)-(c) wrinkled or (d) taut. The 
relaxed strain energy density for $T$ is

$$
W_{f}^{*}(T)=\frac{h E}{2} \begin{cases}0 & \text { if } \delta_{1}<0 \text { and } \delta_{2}<0 ; \quad \text { (a) } \\ \delta_{2}^{2} & \text { if } \mu_{1} \leq 0 \text { and } \delta_{2} \geq 0 ; \quad \text { (b) } \\ \delta_{1}^{2} & \text { if } \mu_{2} \leq 0 \text { and } \delta_{1} \geq 0 ; \quad \text { (c) } \\ \frac{\delta_{1}^{2}+\delta_{2}^{2}+2 \nu \delta_{1} \delta_{2}}{1-\nu^{2}} & \text { if } \mu_{1} \geq 0 \text { and } \mu_{2} \geq 0, \quad \text { (d) }\end{cases}
$$

where $E$ and $\nu$ are the Young's modulus and Poisson's ratio of the film, respectively. ${ }^{9}$ The thickness of the material is given by $h$. See Baginski, et. al. ${ }^{9,10}$ for details.

\section{III.D. Tendon Strain Energy}

Let $N_{t}$ be the total number of tendons. The supporting tendons are modeled as linearly elastic strings. Therefore, the relaxed strain energy density in the $m^{\text {th }}$ supporting tendon is

$$
W_{t, m}^{*}= \begin{cases}\frac{1}{2} K_{t, m} \varepsilon_{m}^{2} & \text { if } \varepsilon_{m} \geq 0 \\ 0 & \text { if } \varepsilon_{m}<0\end{cases}
$$

where $K_{t, m}$ and $\varepsilon_{m} m=1, \ldots, N_{t}$ are the tendon stiffness constant and strain of the tendon, respectively. ${ }^{9}$ The strain in the tendon is calculated by

$$
\varepsilon_{m}=\frac{\Gamma_{m}-\Gamma_{R, m}}{\Gamma_{R, m}}
$$

where $\Gamma_{m}$ and $\Gamma_{R, m}$ are the lengths of the $m^{\text {th }}$ tendon in the deformed and reference configurations, respectively. The initial tension in the supporting tendons can be adjusted by foreshortening all of the $\Gamma_{R, m}$ by a percentage $\gamma$. Support loss in a region can be modeled by setting $K_{t, m}=0$ for desired values of $m$. The total energy in the tendons can then be found by calculating

$$
S_{t}^{*}(\nabla \mathbf{x})=\sum_{m=1}^{N_{t}} \int_{0}^{\Gamma_{R, m}} W_{t, m}^{*}(s) d s .
$$

For computational simplicity, the rigid support structure (to which one end of each tendon is attached) is always in the plane $z=0$. 


\section{Flat Panel Constructions}

Since the molding process is an expensive one ${ }^{1}$ there is a cost benefit to using flat construction patterns. Two different construction patterns based on flat sheets of elastic material are considered. One involves flat gores which seam together like slices of a pie (see Figure 3(a)) and the other involves annular bands which are seamed to form the lateral surface of a frustum (see Figure 3(b)).

The reference configuration for the flat gore construction is generated by initially setting vertices along parabolic arcs extending from the vertex to the rim. The arcs fit the desired parabolic shape of the ideal reflector. There is one arc of vertices on the boundary of each pair of adjacent gores. The remaining vertices for the reference configuration are positioned on straight lines between adjacent pairs of these arcs so that each pie slice is a developable surface. One can then generate the triangular mesh of the reference configuration $\Omega_{R}$ with these vertices. The result is a reference configuration that models a surface having zero curvature in the circumferential direction within the panels. The vertices of the ribs used to generate the entire configuration are highlighted in Figure 3(a) as dots over the corresponding vertices. Notice that the distribution and pattern of the triangular mesh is largely the same as the model for the molded antenna of Figure 1. The configurations differ in that a gore of Figure 3(a) is initially a developable surface while a typical "gore" of Figure 1 is initially doubly curved.

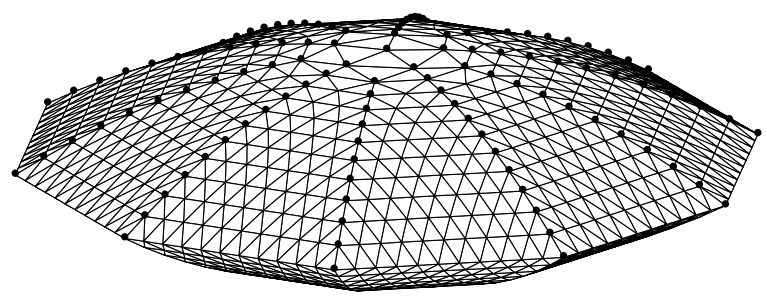

(a) Flat gore construction.

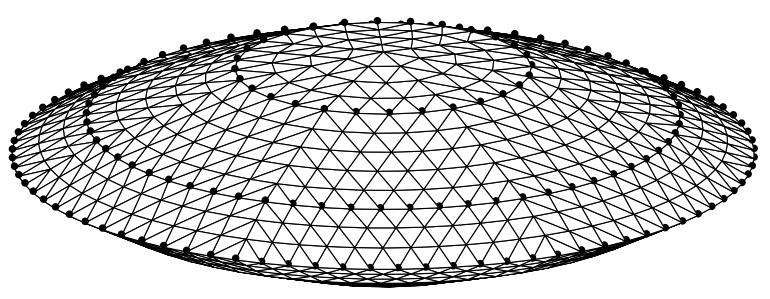

(b) Flat band construction.

Figure 3. Reference configurations with generating ribs for (a) and generating rings for (b).

The other flat construction pattern consists of concentric bands of material which is a surface whose panels have zero curvature in the radial direction but are curved in the circumferential direction. The different panels are modeled in the reference configuration by first fixing rings of vertices to the desired parabolic shape. These are shown by the rings of dots in Figure 3(b). Rows of triangles are then generated between the rings to complete the reference configuration. These bands (on an actual reflector) would be sections of cones and are therefore constructible from flat panels of material. 
It is expected that the flat panel constructions should achieve a nearly parabolic shape when the reflector shell is pressurized. The mesh size of the partition for the flat panels will affect the accuracy of the deformed reflector. These configurations are analyzed in Section VI.

\section{Surface Accuracy and Efficiency Analysis}

For both the molded and flat construction models, one can vary parameters that control the loading forces to study the effect on the surface accuracy of the reflector. The techniques of this research provide the analytical means to assist the design engineer in the selection of appropriate materials, cutting patterns, and support structures that can reduce surface distortions. The accuracy of the deformed reflector can be evaluated by considering the global RMS surface errors as determined by both vertical displacement of the reflector and path length error.

\section{V.A. RMS Calculations}

It is necessary to first establish some notation for the discretized reflector. Denote the centroid of $T_{n} \in \Omega_{R}$ by $\mathbf{p}_{n}$. The corresponding triangle in $\Omega$ is $\mathcal{T}_{n}=\mathbf{x}\left(T_{n}\right)$. Let the centroid of $\mathcal{T}_{n}$ be denoted by $\hat{\mathbf{x}}_{n}=\left(x_{n}, y_{n}, z_{n}\right)$.

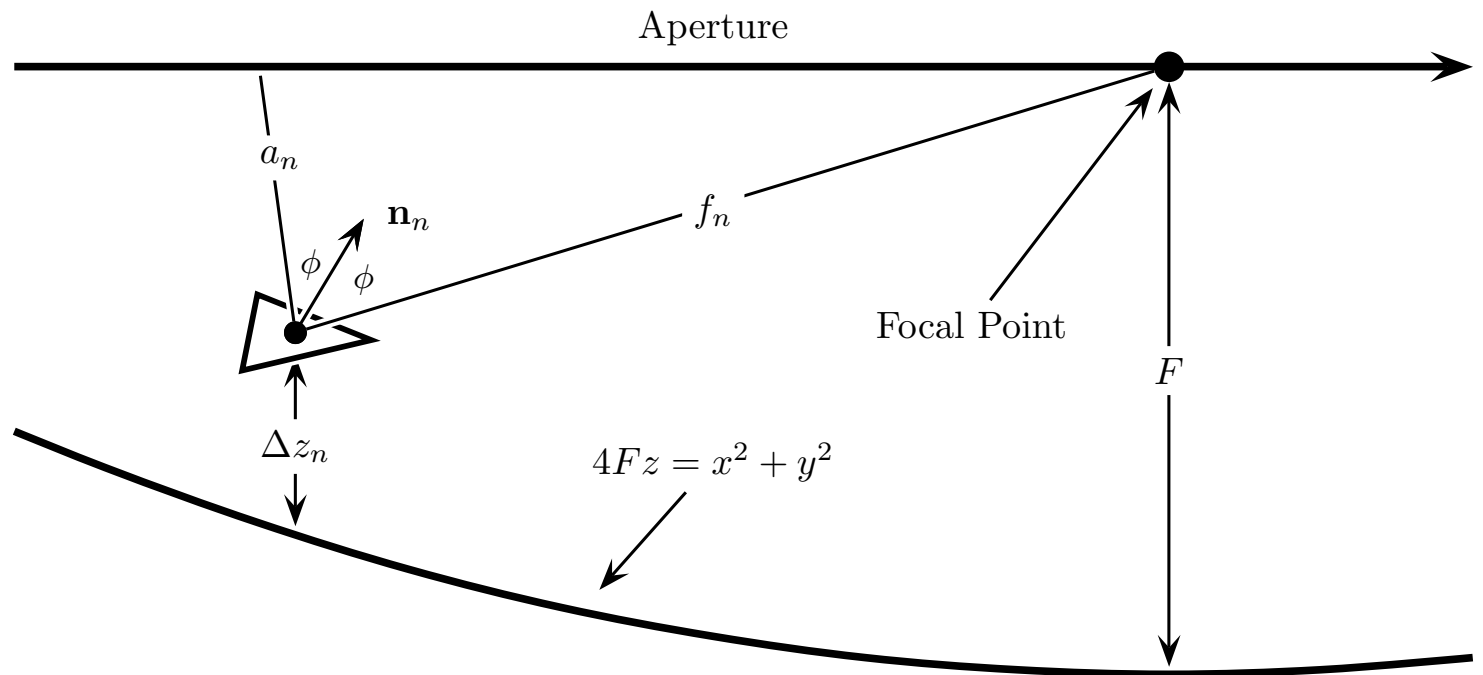

Figure 4. Deformed triangle $\mathcal{T}_{n}$ with centroid $\left(x_{n}, y_{n}, z_{n}\right)$. The vertical displacement, $\Delta z$, is labeled and the pathlength for this triangle is $\ell_{n}=f_{n}+a_{n}$.

A reflector in an ideal position should have each triangle's centroid nearly satisfying the generating equation $4 F z_{n}=x_{n}^{2}+y_{n}^{2}$. The centroids will likely not satisfy the generating curve exactly since it was the vertices of the triangles that were positioned on the parabolic form. For a fine mesh, however, this error is 
negligible. ${ }^{8}$ The Euclidean measure of RMS, $\varepsilon_{\mathrm{EuC}}$, is defined by

$$
\varepsilon_{\mathrm{EUC}}^{2}(\Omega)=\iint_{A}(\Delta z)^{2} \frac{d a}{|A|}=\sum_{n=1}^{N_{f}} \frac{(\Delta z)_{n}^{2}\left|\mathcal{T}_{n}\right|\left(\hat{\mathbf{n}}_{n} \cdot \mathbf{k}\right)}{|A|}
$$

where $\Delta z$ is the vertical displacement between the deformed reflector and an ideal paraboloid, $|A|$ is the area of the circular aperture, $A, d a$ is Lebesgue area measure and $\left|\mathcal{T}_{n}\right|(\hat{\mathbf{n}} \cdot \mathbf{k})$ is the area of $\mathcal{T}_{n}$ projected onto the aperture. ${ }^{5}$

Another RMS calculation includes the pathlength error of a radiation ray traveling from the focal point to the focal plane via triangle $\mathcal{T}_{n}$. For an ideal paraboloid, this distance should be twice the focal length of the parabolic reflector for all rays of radiation. Having denoted the total path length of a ray striking the centroid of $\mathcal{T}_{n}$ by $\ell_{n}$, one gets

$$
\varepsilon_{\mathrm{RMS}}^{2}(\Omega)=\iint_{A}\left(\frac{\Delta \ell}{2}\right)^{2} \frac{d a}{|A|}=\sum_{n=1}^{N_{f}} \frac{\left(\ell_{n}-2 F\right)^{2}\left|\mathcal{T}_{n}\right|\left(\hat{\mathbf{n}}_{n} \cdot \mathbf{k}\right)}{4|A|}
$$

where $\Delta \ell=\ell_{n}-2 F$ is the pathlength error of a ray reflected at a given point on the reflector surface and $F$ is the focal distance of the reflector. ${ }^{5}$

\section{V.B. Antenna Gain and Efficiency Calculations}

It is desirable to consider the antenna's efficiency by computing the far zone electric and magnetic fields that are reflected by the antenna's reflector surface. The analysis applied here closely follows the computation methods in Chapter 8 of Stutzman and Thiele. ${ }^{12}$ In the following equations, the permeability and permittivity of free space are $\mu_{0}=4 \pi \times 10^{-7} \mathrm{~N} / \mathrm{Amp}^{2}$ and $\varepsilon_{0}=8.854 \times 10^{-12}$ coul $^{2} / \mathrm{N} \mathrm{m}^{2}$, respectively. ${ }^{13}$ This model uses a simple dipole for the feed having current $I_{0}=200$ Amps oriented along the $y$-axis with dipole length $\Delta y=1 \mathrm{~cm}$. In this case, the incident magnetic field on the reflector is

$$
\mathbf{H}_{\mathrm{INC}}=-i \frac{I_{0} \beta \Delta y}{4 \pi r^{\prime}} e^{-i \beta r^{\prime}}\left(\hat{\mathbf{r}}^{\prime} \times \hat{\mathbf{j}}\right)
$$

where $\hat{\mathbf{r}}^{\prime}$ is the unit direction vector from the focus to a point on the reflector, $\beta^{2}=\omega^{2} \varepsilon_{0} \mu_{0}$ and $i=\sqrt{-1}$. Also, $\omega=2 \pi f$ is the angular frequency associated with $f$. The far zone electric and magnetic fields can be determined from the magnetic vector potential $\mathbf{A}$ by

$$
\mathbf{E}=-i \omega \mu_{0}(\mathbf{A}-(\hat{\mathbf{r}} \cdot \mathbf{A}) \hat{\mathbf{r}}) \quad \text { and } \quad \mathbf{H}=(\hat{\mathbf{r}} \times \mathbf{E}) / \eta
$$


where $\eta^{2}=\mu_{0} / \varepsilon_{0}$ is the impedance of free space. The power per steradian in any given direction from the antenna (in spherical coordinates) can be obtained from the reflected electric field $\mathbf{E}$ and magnetic field $\mathbf{H}$ by the Poynting Vector in the relation

$$
U(\theta, \phi)=r^{2}\left(\mathbf{E} \times \mathbf{H}^{*}\right) \cdot \hat{\mathbf{r}} .
$$

The function $U$ is the radiation intensity in a given polar direction $(\theta, \phi)$ where the antenna is positioned with its vertex at the origin. This intensity function is independent of the distance from the antenna since

$$
\mathbf{E} \times \mathbf{H}^{*} \sim r^{-2} .
$$

A parabolic reflector should yield a radiation pattern $U$ which achieves its maximum in the direction the antenna is pointed (for this case $\theta=\phi=0$ ). Denote by $U_{m}$ and $U_{m}^{p}$ the maximum radiation intensity for the deformed faceted surface configuration and an ideal paraboloid of the same dimensions, respectively. Then, the component of the efficiency due to reflector surface distortions is

$$
e_{s}=U_{m} / U_{m}^{p}
$$

The results in this paper include efficiency computations that incorporate the trade off between the spill-over and under-illumination efficiency calculations. The maximum efficiency of any parabolic reflector can be no more than $82 \%$ or the value in Equation (9). ${ }^{12}$ The effective efficiency $e^{*}$ is defined as

$$
e^{*}=0.82 e_{s}
$$

Besides this tradeoff and surface distortions, no other efficiency losses are considered. The ideal and effective antenna gain are found by,

$$
G_{I}=4 \pi A / \lambda^{2} \quad \text { and } \quad G=e^{*} G_{I},
$$

respectively. Note that $\lambda=c / f$ where $c$ is the speed of light. Unless otherwise noted, the data presented for gain and efficiency are calculated for an RF value of $f=40 \mathrm{GHz}$.

\section{Numerical Results}

This section consists of results which model reflectors having axisymmetric reference configurations. The material properties of Kapton are used to serve as the reflector material and the dimensions of an actual prototype are applied. Table 1 contains parameter values that are used in the following numerical experi- 
ments. In certain instances, the dimensions of Table 1 are scaled in order to study the effect of the size and depth of a reflector on antenna performance.

Table 1. Mechanical properties of Kapton, reflector dimensions and grid specifications.

\begin{tabular}{lcc}
\hline \hline Property & Variable & Value \\
\hline Kapton Thickness & $h$ & $12.7 \mathrm{microns}$ \\
Kapton Mass Density & $\rho$ & $1.42 \mathrm{~g} / \mathrm{m}^{3}$ \\
Kapton Young's Modulus & $E$ & $2.59 \mathrm{GPa}$ \\
Kapton Poisson's Ratio & $\nu$ & 0.34 \\
\hline Reference Tendon Length & $\Gamma_{R, m}$ & $6.00 \mathrm{~cm}$ \\
Reflector Diameter & $D$ & 2.13 meters \\
Reflector Focal Length & $F$ & 0.914 meters \\
\hline Total Number of Facets & $N$ & 20,736 \\
Number of Reflector Facets & $N_{f}$ & 10,368 \\
Number of Supporting Tendons & $N_{t}$ & 72 \\
\hline \hline
\end{tabular}

Previous numerical experiments ${ }^{6,8}$ demonstrated that the elastic reflector easily deforms when the force of the supporting tendons is larger than approximately $0.5 \mathrm{~N}$. For example, a force of $2.304 \mathrm{~N}$ corresponded to a 2 to 3 degree deflection near the rim of the reflector and a vertical displacement of approximately 4 mm near the vertex. ${ }^{6}$ This sort of deformation can cause the antenna to suffer a loss of power gain, particularly at higher radio frequencies.

Some earlier results ${ }^{7}$ suggest that the antenna performance can be improved by increasing the number of gores in the flat panel construction. This initial work also seemed to suggest there was a preferred setting for the differential pressure, $p_{0}$. Too low a pressure allowed wrinkling in the deformed reflector while too high a pressure caused excessive bulging of the reflector membrane.

Two studies are presented here which focus on the behavior of the reflector's edge. The first entails a modification to the flat band construction pattern of Section IV. The other consists of fixing the rim of the reflector. Both experiments were conceived in an attempt to mitigate deflection of the reflector near the rim. ${ }^{7}$ Parametric studies conducted for an antenna of diameter $D=2.13$ meters will be presented in Sections VI.B and VI.C. An analysis of a large diameter antenna ( $D=8.52$ meters) is also presented.

\section{VI.A. Reflector Size Variation}

This section contains an examination of the relationship between the reflector's size and the surface accuracy of the antenna when other significant parameters are held constant. Two case studies are performed; one 
with a tendon foreshortening of $\gamma=0.03$ and the other with $\gamma=0.06$. For each case study, the size of the reflector is increased while holding the ratio $F / D=0.4291$. The reflector sizes in Table 2 are obtained by scaling the reflector of Table 1 by factors of 1.0, 2.5, 4.0, 5.5 and 7.0. The tendon lengths are not changed by the size factor. Gravity is held at $\mathbf{g}=-g \mathbf{k}$ for these tests and $p_{0}=10$ Pa. The equilibrium configuration is determined by the model for these parameters and the surface accuracy data is reported in Table 2 .

Table 2. Comparison of efficiency for various antenna sizes and supporting conditions.

\begin{tabular}{cc|rrc|rrc}
\hline \hline \multicolumn{2}{|c|}{ Reflector Dimensions } & \multicolumn{3}{|c|}{ Case I: $\gamma=0.03$} & \multicolumn{3}{c}{ Case II: $\gamma=0.06$} \\
$D(\mathrm{~m})$ & $F(\mathrm{~m})$ & $\varepsilon_{\mathrm{RMS}}(\mathrm{mm})$ & $e^{*}$ & Gain dB & $\varepsilon_{\mathrm{RMS}}(\mathrm{mm})$ & $e^{*}$ & Gain dB \\
\hline 2.130 & 0.914 & 1.4156 & $80.84 \%$ & 58.09 & 4.2595 & $74.91 \%$ & 57.75 \\
5.325 & 2.285 & 4.7162 & $76.65 \%$ & 65.81 & 2.5938 & $80.31 \%$ & 66.02 \\
8.520 & 3.656 & 15.5799 & $50.77 \%$ & 68.11 & 14.5017 & $54.82 \%$ & 68.44 \\
11.715 & 5.027 & 31.0087 & $39.64 \%$ & 69.80 & 28.1322 & $43.64 \%$ & 70.22 \\
14.910 & 6.398 & 50.8096 & $29.91 \%$ & 70.67 & 52.2116 & $33.49 \%$ & 71.16 \\
\hline \hline
\end{tabular}

By comparing the results of Cases I and II in Table 2, one can see that the size of the reflector having greatest efficiency for Case I is different from that of Case II. This is not surprising since the mass of the reflector is increased by the square of the scaling factor. A greater supporting force in the tendons is likely necessary to counter the larger mass. Specific guidelines would depend on the particular application and the surface accuracy tolerances.

For Case II, the antenna power gain is included. One can still attain higher levels of power gain for larger diameter antennas despite the reduction of efficiency. From Equation (10), it can be found that the antenna in case $(D, \gamma)=(14.91,0.06)$ performs like an antenna operating at $100 \%$ efficiency with diameter 8.63 meters. While larger aperture antennas are desired for increased power transmission, higher accuracy will be required to make use of the full size of the reflector.

\section{VI.B. Parametric Study of Edge Forces and Reflector Shape Modification}

Since one serious problem with an inflatable antenna is the deflection of the reflector near the rim, the following parametric study that involves modifying the reflector rim shape is conducted. The band construction is well-suited for this experiment since the rim of the reflector is contained in one section of the cutting pattern. The slope of this outer most band is modified by decreasing the radius of the outer most ring of vertices (situated at the reflector's rim) as shown in Figure 5. 


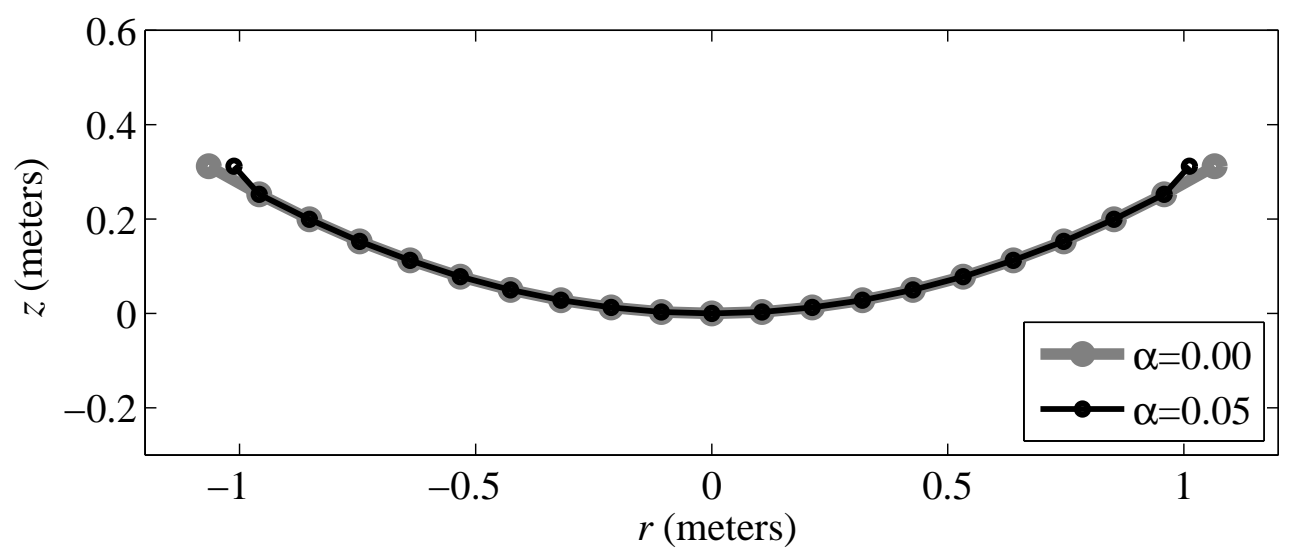

Figure 5. Generating curve for a flat ring configuration with $\alpha=0.05$.

The band construction in this parametric study consists of 10 bands with the seams being set at intervals of $0.1 R_{f}$ where $R_{f}=\frac{D}{2}$ is the radius of the aperture. A shape modification factor $\alpha \in[0,1]$ is introduced to adjust the outer most band. This factor determines the radial distance, $d$, from the vertex of the paraboloid to the outer most ring of vertices. In particular, $d=(1-\alpha) R_{f}$. Note that the reference configuration is unchanged for $\alpha=0$. The result is a reference configuration modeling a paraboloid for the inner nine bands with a tapered adjustment for the tenth band.

The geometric adjustment to the rim of the reference configuration was introduced to explore means of adjusting the construction patterns in order to help reduce the deflection due to the tension in the supporting tendons. A set of tests was performed to determine the reflector's shape accuracy as a function of the rim radius modification factor, $\alpha$. The differential pressure is set to $p_{0}=12.5 \mathrm{~Pa}$ and the acceleration due to gravity is set at $\mathbf{g}=-g \mathbf{k}$. Table 3 contains $\varepsilon_{\text {RMS }}$ values as calculated by Equation (8) for various values of the parameters $\alpha$ and $\gamma$.

(a) $D=2.13$ meters

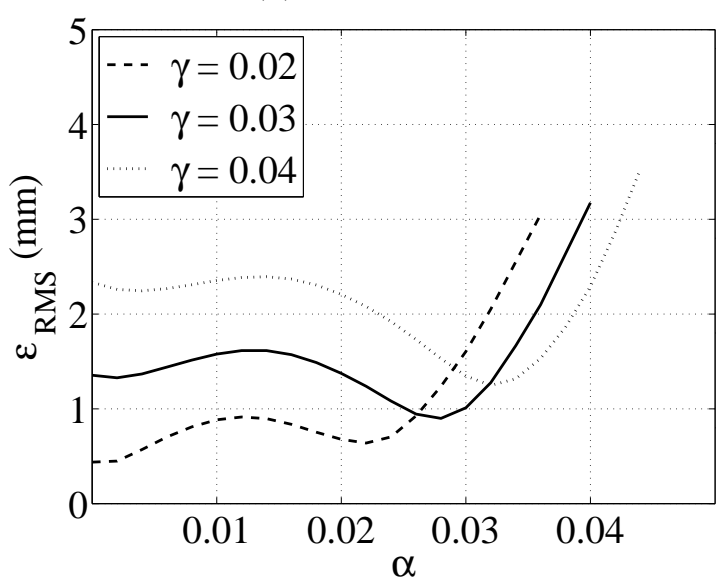

(b) $D=8.52$ meters

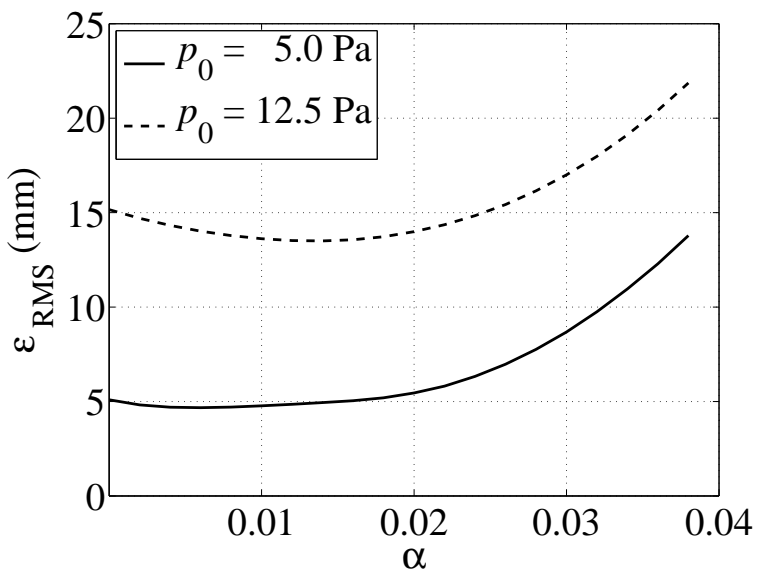

Figure 6. Effect of rim modification factor on $\varepsilon_{\mathrm{RMS}}$ for a small(a) and large(b) inflatable antenna. 
For each value of $\gamma$, the strain energy in the tendons is determined by using Equation $(5)$ where $K_{t, m}=$ $250 \mathrm{~N}$ for all $N_{t}$ tendons. In Figure 6(a), $\varepsilon_{\mathrm{RMS}}$ is plotted as a function of $\alpha$ for $0 \leq \alpha \leq 0.044$ and for $\gamma=0.01,0.02,0.03$ and 0.04. One can see in Figure $6(\mathrm{a})$ that $\varepsilon_{\mathrm{RmS}}$ can be reduced by selecting $\alpha$ appropriately. For fixed $\gamma$, one can find a parameter value $\alpha=\alpha_{\gamma}^{*}$ such that

$$
\varepsilon_{\mathrm{RMS}}\left(\alpha_{\gamma}^{*}, \gamma\right)=\min _{\alpha \in A} \varepsilon_{\mathrm{RMS}}(\alpha, \gamma)
$$

where $A=\{\alpha \mid 0 \leq \alpha \leq 4.4\}$. Results concerning these local minima are summarized in Table 3. Columns four through six contain the data for the flat band reflector that achieves the minimal $\varepsilon_{\text {RMS }}$ value for each $\gamma$. The gain is calculated as in Equation (10). The second and third columns of Table 3 contain the $\varepsilon_{\text {RMs }}$ attained by the molded reflector construction and the unmodified flat band construction $(\alpha=0)$, for comparison. The reduction in $\varepsilon_{\mathrm{RMS}}\left(\right.$ seventh column) compares the values $\varepsilon_{\mathrm{RMS}}(0, \gamma)$ and $\varepsilon_{\mathrm{RMS}}\left(\alpha_{\gamma}^{*}, \gamma\right)$.

Table 3. Comparison of $\varepsilon_{\mathrm{RMS}}(\alpha, \gamma)$ values $(\mathbf{m m})$ for various $\gamma$. Aperture diameter is $D=2.13$ meters. Reduction in $\varepsilon_{\mathrm{RMS}}$ is based on a comparison of Column 3 and Column 5.

\begin{tabular}{c|cc|ccc|c}
\hline \hline$\gamma$ & Molded & $\alpha=0$ & \multicolumn{3}{|c|}{ Reflector with $\alpha=\alpha_{\gamma}^{*}$} & Reduction \\
& $\varepsilon_{\mathrm{RMS}}$ & $\varepsilon_{\mathrm{RMS}}(0, \gamma)$ & $\alpha_{\gamma}^{*}$ & $\varepsilon_{\mathrm{RMS}}\left(\alpha_{\gamma}^{*}, \gamma\right)$ & Gain $(\mathrm{dB})$ & in $\varepsilon_{\mathrm{RMS}}$ \\
\hline 0.01 & 0.8082 & 0.5084 & 0.002 & 0.4718 & 58.11 & $7.2 \%$ \\
0.02 & 0.2238 & 0.4369 & 0.000 & 0.4369 & 58.13 & - \\
0.03 & 1.0456 & 1.3537 & 0.028 & 0.8993 & 56.26 & $33.6 \%$ \\
0.04 & 1.9960 & 2.3340 & 0.032 & 1.2478 & 55.95 & $46.5 \%$ \\
\hline \hline
\end{tabular}

The results in Table 3 advocate for the use of design parameters such as $\alpha$ to help reduce surface distortions such as those generated by the boundary support forces. Consider, for example, the tendon foreshortening factor $\gamma=0.03$. The best surface accuracy attained is $\varepsilon_{\mathrm{RMS}}=0.8993 \mathrm{~mm}$ for the parameters $(\alpha, \gamma)=(0.028,0.03)$. This $\varepsilon_{\mathrm{RMS}}$ value is $33.6 \%$ less than that of the case $(\alpha, \gamma)=(0,0.03)$. The case for $(\alpha, \gamma)=(0.028,0.03)$ also has an $\varepsilon_{\mathrm{RMS}}$ value that is $14.0 \%$ lower than $\varepsilon_{\mathrm{RMS}}=1.0456 \mathrm{~mm}$ for the molded reflector.

To investigate larger antennas, the analysis with the rim modification factor $\alpha$ is repeated with $D=$ 8.52 meters. In this case, one finds that the tendon foreshortening does not affect $\varepsilon_{\text {RMs }}$ as much as the differential pressure, $p_{0}$. Hence, $\gamma=0.03$ is fixed and the larger antenna tested for several different different pressure levels. In Figure $6(\mathrm{~b})$, note that $\varepsilon_{\mathrm{RMS}}$ can be reduced with a particular value of $\alpha$, as was the case for the smaller antenna. The usual choice of $p_{0}=12.5 \mathrm{~Pa}$, however, does not seem to be preferred in this case. The data of this study suggests that changing the conditions from $\left(\alpha, p_{0}\right)=(0.014,12.5)$ to $\left(\alpha, p_{0}\right)=(0.006,5)$ will lead to a reduction in $\varepsilon_{\mathrm{RMS}}$ by $65.4 \%$. For comparison with the plots of Fig- 
ure $6(\mathrm{~b})$, note that the ideal configuration (such as seen in Figure 1) for an antenna of this size would have $\varepsilon_{\mathrm{RMS}}=0.4256 \mathrm{~mm}$. This datum is based on the ideal reflector geometry and is independent of the differential pressure, $p_{0}$.

A practical consideration to reduce the rim effect entails reducing the area of the elastic reflector that is treated for reflectivity. In particular, one can neglect treating the outer edge of the reflector, where surface distortions tend to concentrate. This scenario is modeled by removing the outer most ring of triangular elements from the computations of $U_{m}$ as defined in Section V.B. Recall the case with $\left(D, p_{0}, \alpha\right)=(8.52,12.5,0.014)$ which resulted in $\varepsilon_{\mathrm{RMS}}=13.5066 \mathrm{~mm}$ and $G_{\mathrm{dB}}=66.82$. For this case, neglecting the outermost ring of triangles is equivalent to treating 2.30 square meters less of the surface for reflectivity (the total reflector surface for this case is 57.86 square meters). Note that this neglected area is only a portion of the outer most band of the flat band construction. The result obtained yields $\varepsilon_{\mathrm{Rms}}=13.3248 \mathrm{~mm}$ and $G_{\mathrm{dB}}=67.99$. Despite a decrease in the radiating aperture, removal of the distorted reflector edge from consideration allowed the gain to increase slightly.

In both the case of a large and small antenna, the rim modification factor is effective in reducing $\varepsilon_{\mathrm{RmS}}$. Other physical factors must be considered to help further the reduction of $\varepsilon_{\text {RMS }}$. Which physical parameters are most important and to what extent, will require further investigation.

\section{VI.C. Tendon Supported vs. Fixed Boundary Reflectors}

Figure $6(\mathrm{a})$ reveals that for sufficiently large $\gamma$ (in that case $\gamma>0.02), \varepsilon_{\mathrm{RMS}}\left(\alpha_{\gamma}^{*}, \gamma\right)$ increases as a function of $\gamma$. This suggests that the surface accuracy may inevitably suffer from very strong tendon forces. This motivates an investigation of the antenna's $\varepsilon_{\text {RMS }}$ when the reflector is not subject to tendon forces at the boundary. In this section, the antenna reflector is modeled by using the flat band construction, fixing the boundary to a rigid frame, and setting $\alpha=0$. This entails eliminating $S_{t}^{*}$ from Equation (2) and fixing the vertices of the antenna's rim. Equilibrium shapes are calculated for the differential pressures $p_{0}=5,10,15$, 20, and $25 \mathrm{~Pa}$. The shapes are also tested in three different gravitational fields as listed in Table 4.

Table 4. Values of $\varepsilon_{\mathrm{RMS}}\left(p_{0}, \mathrm{~g}\right)(\mathrm{mm})$ for a fixed boundary reflector with $D=2.13$ meters.

\begin{tabular}{cccccc}
\hline \hline Gravity & $p_{0}=5 \mathrm{~Pa}$ & $p_{0}=10 \mathrm{~Pa}$ & $p_{0}=15 \mathrm{~Pa}$ & $p_{0}=20 \mathrm{~Pa}$ & $p_{0}=25 \mathrm{~Pa}$ \\
Vector: $\mathbf{g}$ & $\varepsilon_{\mathrm{RMS}}\left(p_{0}, \mathbf{g}\right)$ & $\varepsilon_{\mathrm{RMS}}\left(p_{0}, \mathbf{g}\right)$ & $\varepsilon_{\mathrm{RMS}}\left(p_{0}, \mathbf{g}\right)$ & $\varepsilon_{\mathrm{RMS}}\left(p_{0}, \mathbf{g}\right)$ & $\varepsilon_{\mathrm{RMS}}\left(p_{0}, \mathbf{g}\right)$ \\
\hline $\mathbf{0}$ & 0.1923 & 0.0853 & 0.2483 & 0.4454 & 0.6450 \\
$-g \mathbf{k}$ & 0.1861 & 0.0879 & 0.2546 & 0.4519 & 0.6516 \\
$-g \mathbf{j}$ & 0.1925 & 0.0856 & 0.2484 & 0.4454 & 0.6450 \\
$-g(\mathbf{j}+\mathbf{k}) / \sqrt{2}$ & 0.1969 & 0.0807 & 0.2447 & 0.4420 & 0.9556 \\
\hline \hline
\end{tabular}


Generally, the shape of the reflector is far more accurate for these experiments than for tests involving the supporting tendons. For the pressure values of 10 and $15 \mathrm{~Pa}$, the fixed boundary reflector models have surface accuracy range $0.0853 \leq \varepsilon_{\mathrm{RMS}} \leq 0.2546$ as presented in Table 4 . Table 3 shows that the band construction achieves its best surface accuracy of $\varepsilon_{\mathrm{RMS}}=0.4369$ for the case $(\alpha, \gamma)=(0,0.02)$. Figure 7 compares the vertical discrepancy of the deformed reflector, as calculated in Equation (7), for both a fixed rim antenna and tendon-supported one. Each data point in Figure 7 plots the average vertical facet displacements of the facets in each band against the outermost radial extent of the band. The bars surrounding the data points represent the spread of the displacement data for the given band. With exception of the boundary support and configuration type, all conditions for the two tests are identical. The internal pressure is held at 12.5 $\mathrm{Pa}$ and $\mathbf{g}=-g \mathbf{k}$

The top set of data in Figure 7 is for a tendon supported antenna modeled with the band configuration and $(\alpha, \gamma)=(0.028,0.03)$. The lower set of data is for a fixed boundary reflector modeled with the band configuration. The tenth band of the tendon-supported reflector has a large variance in displacement since $\alpha=0.028$ for that case.

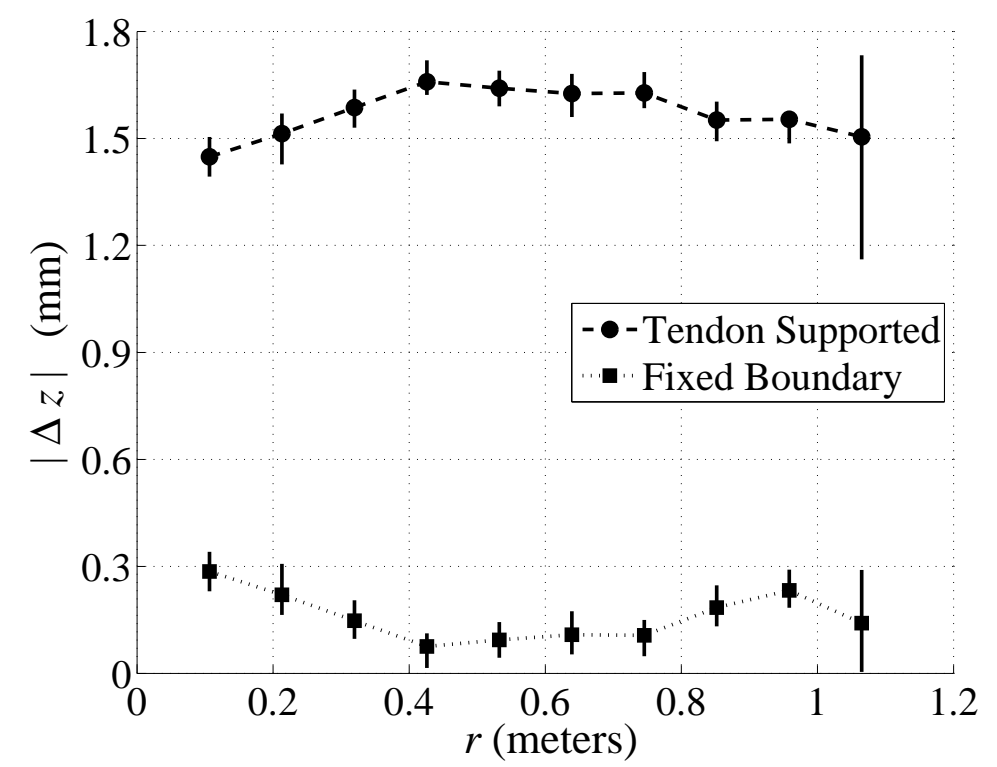

Figure 7. Comparison of error for a fixed rim and a tendon-supported reflector.

The low RMS values for the fixed boundary reflector suggest good performance at radio frequencies greater than $40 \mathrm{GHz}$. It is clear that the $\varepsilon_{\mathrm{RMS}}$ values, despite their growth for larger pressure values, remain low compared to the $\varepsilon_{\text {RMS }}$ values of the study shown in Table 3 . Changes to the gravity field have little affect on the $\varepsilon_{\mathrm{RMS}}$ values. The radiation and efficiency data in Table 5 show that the performance of a fixed boundary antenna may be able to perform well at frequencies above Ka-Band. 
Table 5. Characterization of fixed rim inflatable antennas; $p_{0}=12.5 \mathrm{~Pa}$.

\begin{tabular}{lcccc}
\hline \hline Flat Band Construction, $\alpha$ & 0.000 & 0.000 & 0.000 & 0.028 \\
Edge Condition & Fixed Rim & Fixed Rim & Fixed Rim & $\gamma=0.03$ \\
\hline Radiation Frequency, $f(\mathrm{GHz})$ & 40 & 60 & 80 & 80 \\
Intensity at Boresight (Watts/Str) & 1282.71 & 2884.89 & 5125.71 & 3004.45 \\
Antenna Gain (dB) & 58.1427 & 61.6630 & 64.1591 & 60.9769 \\
Side Lobe Level - H plane (dB) & -17.57 & -17.59 & -17.56 & -17.29 \\
Side Lobe Level - E plane (dB) & -24.19 & -24.18 & -24.18 & -21.82 \\
\hline Radiometric RMS (mm) & 0.1509 & 0.1509 & 0.1509 & 0.8993 \\
Euclidean RMS (mm) & 0.1641 & 0.1641 & 0.1641 & 4.1233 \\
Efficiency, $e^{*}$ & $81.91 \%$ & $81.88 \%$ & $81.83 \%$ & $47.96 \%$ \\
\hline \hline
\end{tabular}

At a radio frequency level of $80 \mathrm{GHz}$, the fixed rim antenna is much more efficient than the tendon supported one; see Table 5 . Note that the tendon supported reflector has an $\varepsilon_{\mathrm{RMS}}$ on the order of $1 \mathrm{~mm}$. For high radio frequency, however, the $\varepsilon_{\mathrm{RMS}}$ has far lower tolerance. If large reflector antennas are used for high frequency applications, one may wish to re-examine the boundary support.

Table 6. Values of $\varepsilon_{\mathrm{RMS}}\left(p_{0}, \mathbf{g}\right)(\mathbf{m m})$ for a reflector with $D=8.520$ meters.

\begin{tabular}{lccccc}
\hline \hline Gravitational & $p_{0}=5 \mathrm{~Pa}$ & $p_{0}=10 \mathrm{~Pa}$ & $p_{0}=15 \mathrm{~Pa}$ & $p_{0}=20 \mathrm{~Pa}$ & $p_{0}=25 \mathrm{~Pa}$ \\
Environment & $\varepsilon_{\mathrm{RMS}}\left(p_{0}, \mathbf{g}\right)$ & $\varepsilon_{\mathrm{RMS}}\left(p_{0}, \mathbf{g}\right)$ & $\varepsilon_{\mathrm{RMS}}\left(p_{0}, \mathbf{g}\right)$ & $\varepsilon_{\mathrm{RMS}}\left(p_{0}, \mathbf{g}\right)$ & $\varepsilon_{\mathrm{RMS}}\left(p_{0}, \mathbf{g}\right)$ \\
\hline $\mathbf{g}=\mathbf{0}$ & 1.8725 & 5.0434 & 8.1638 & 11.2398 & 14.2787 \\
$\mathbf{g}=-g \mathbf{k}$ & 1.7677 & 4.9387 & 8.0599 & 11.1368 & 14.1764 \\
\hline \hline
\end{tabular}

From Figure $6(\mathrm{~b})$, recall that $\varepsilon_{\mathrm{RMS}}$ for the larger antenna ( $D=8.520$ meters) has greater dependence on $p_{0}$ than on $\gamma$. The rim is fixed for this reflector and the same test is performed as was for the smaller fixed rim reflector. The data in Table 6 shows that the surface distortions are not confined to sub-millimeter quantities for the larger antenna. This data does confirm that the pressure term has a substantial effect on $\varepsilon_{\mathrm{RMS}}$. While a fixed rim may be a good solution for a reflector with a diameter of approximately 2 meters, the model does not suggest the fixed rim for an antenna with a diameter of nearly 8 meters.

\section{VI.D. Parametric Study of Reflector Depth}

The results in this section suggest that the depth of an antenna reflector is an important factor affecting the antenna's performance. Since the antenna that is used for most of the experiments has $F / D=0.4291$, the reflector is tested with multiples of this value. First, a base value of $F$ is determined depending on the reflector diameter, $D$. In the case $D=2.13$ meters, the base value $F_{b}=0.914$ meters is obtained. Then, various $F / D$ ratios are tested by substituting the values $\left\{0.25 F_{b}, 0.5 F_{b}, 0.75 F_{b}, \ldots, 3.0 F_{b}\right\}$ for the focal length. 
For this experiment, the pressure is set at $12.5 \mathrm{~Pa}$ and the tendon foreshortening to $\gamma=0.03$. See Figure 8. The antenna reflector having $D=2.13$ meters, holds an efficiency $e^{*}$ of at least $80.16 \%$ for all the $F / D$ values tested. For the case, $D=5.325$ meters, the efficiency drops substantially as the $F / D$ ratio increases. At $F / D=1.2873$, the model predicts a performance efficiency of only $e^{*}=4.08 \%$. An even faster reduction of the antenna performance is seen for the large antenna of $D=10.650$ meters. In that case, $e^{*}=0.30 \%$ is obtained when $F / D=1.2873$. Additional data points for antennas with aperture diameters of 3.195 and 3.728 meters are also plotted. This data shows that the efficiency of mid-size antennas is also compromised when $F / D$ grows, although less severely.

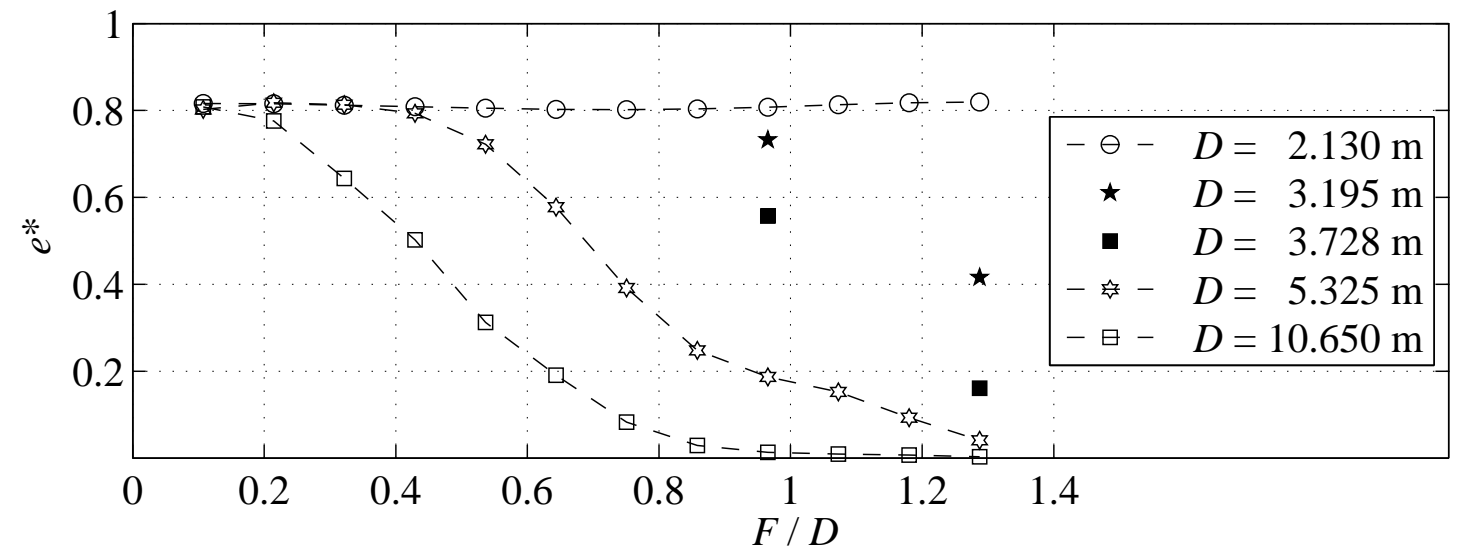

Figure 8. Antenna performance for various $F / D$ values and antenna sizes.

Finally, consider the case with $(F / D, D, \gamma)=(1.2873,5.325,0.03)$ where $e^{*}=4.08 \%$ was found. A small improvement, $e^{*}=7.81 \%$, is achieved when a fixed boundary is applied (see Section VI.C). Upon further examination of the fixed rim boundary condition for a shallow reflector, one finds that the reflector is displaced by approximately $2.5 \mathrm{~cm}$. For an equivalent tendon supported reflector, the vertex of the reflector was displaced by approximately $4.2 \mathrm{~cm}$. Since the two parabolic sheets are joined together at a shallow angle for these antennas, there is tendency for the two sheets to separate after pressurizing; in other words, "bulging" is found to occur near the rim.

It is expected that the stress resultants will increase in the case of a shallow reflector. The Laplace-Young Equation asserts that

$$
p_{0}=\sigma_{1} k_{1}+\sigma_{2} k_{2}
$$

where $\sigma_{i}$ are the principal stress resultants and $k_{i}$ are the principal curvatures. For a fixed differential pressure $p_{0}$, the $\sigma_{i}$ must increase as the principle curvatures decrease. 
(a) $D=2.13$ meters

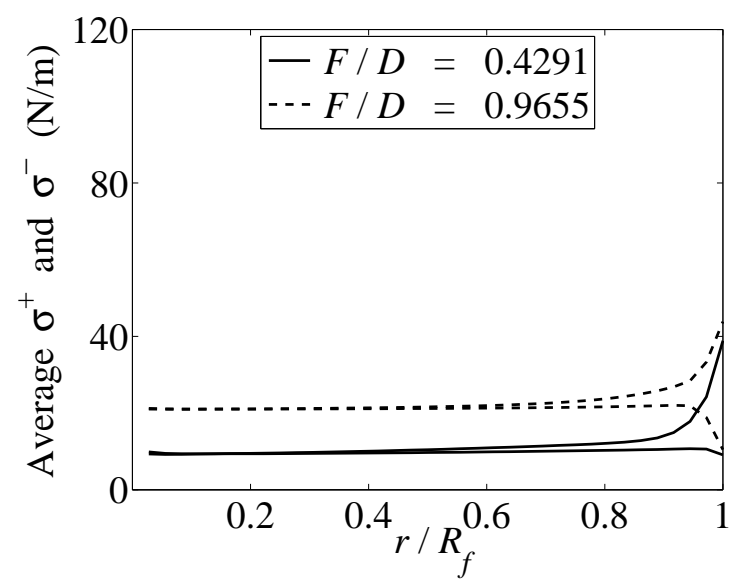

(b) $\quad D=10.65$ meters

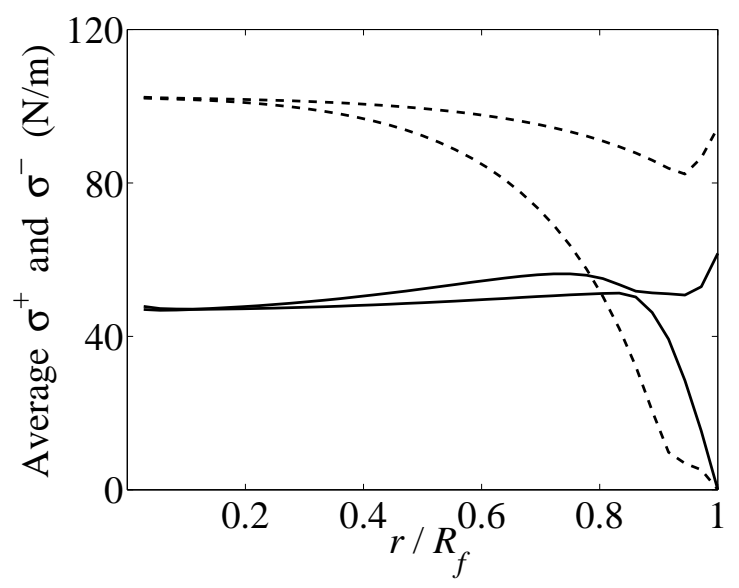

Figure 9. Antenna performance for various $F / D$ values and antenna sizes.

In Figure 9, the stress resultants for four antenna cases are plotted. Since the stress distribution of the deformed reflector is nearly radially symmetric in these cases, the principle stress resultants are plotted as a function of radial distance from the vertex. The solid lines of Figure 9 indicate a deeper reflector while the dotted lines are for a shallower reflector. For each case, two lines are plotted to show the average maximum stress $\left(\sigma^{+}(r)\right)$ and average minimum stress $\left(\sigma^{-}(r)\right)$. The plots indicate that the stress resultants at the vertex of the reflector are nearly twice the magnitude for the shallow reflector versus the deeper one. This holds true for both the smaller and larger diameter reflectors. Also note that $\sigma^{-}$nears zero for the larger shallow reflector. This suggests an increase likelihood of wrinkling near the rim of the reflector, especially since the maximum stress resultant $\sigma^{+}$remains high around $90 \mathrm{~N} / \mathrm{m}$.

\section{Conclusions}

Large aperture reflectors are able to radiate more energy than smaller reflectors. However, the results of this paper show that larger reflectors typically are not able to attain sub-millimeter RMS values. For this reason, large antennas exhibit a trade off between the ideal gain that could be achieved with a large aperture and the effective antenna gain which is likely reduced due to surface distortions.

Flat construction patterns, in certain cases, may lead to lower fabrication costs and simplification of the construction method. Furthermore, the rim modification factor can be used to reduce $\varepsilon_{\text {RMS }}$. In the case of a large size antenna, additional factors must also be considered. These results suggest that the surface accuracy has greater dependence on the differential pressure than on the rim modification parameter when 
the reflector diameter is of order 10 meters. This is in contrast to a smaller antenna, where the supporting tendons have a greater effect on surface accuracy. One result, however, suggested the possibility of a slight gain increase by not treating the outer edge of the paraboloid for reflectivity.

The support tendons are the principle factor that distorts the 2.13 meter reflector from the desired parabolic shape. A fixed reflector boundary leads to a substantial reduction of the RMS values. Variations in the direction and strength of gravity as well as the internal pressure do not cause overall RMS values greater than $1 \mathrm{~mm}$. Unfortunately, elimination of the supporting tendons may lead to deployment problems with a different support structure. Also, the model for a larger antenna does not perform as well with a fixed boundary. The RMS values do not remain below $1 \mathrm{~mm}$ and it is clear that an increase in the differential pressure could cause a deformation that negates the benefits of a fixed rim.

Many of the results presented here point to key design parameters that can be tuned to increase the performance of inflatable parabolic reflectors. Further analysis on large reflectors will need to be carried out in order to characterize their behavior for different operating environments. However, the analytical methods developed here show promise that with additional analysis a more complete picture of large aperture reflector behavior can be attained.

\section{References}

1 Romanofsky, Robert R., Kevin Lambert, Brian W. Welch and Irene Bibyk. "The Potential for Gossamer Deployable Antenna Systems in Ka-Band Exploration and Science Communications Architectures." Twelfth Ka and Broadband Communications Conference. Napoli, Italy. September 26-27 2006.

${ }^{2}$ Freeland, R. E., G. Bilyeu, G.R. Veal and M.M. Mikulas. "Inflatable Deployable Structures Technology Summary." International Astronautical Federation, Paper IAF-98-I.5.01, September - October 1998.

3 Thomson, Mark W. "Astromesh Deployable Reflectors for Ku and Ka Band Commercial Satellites." AIAA $2002-2032$.

${ }^{4}$ Ruze, John. "Physical Limitations on Antennas." MIT Research Laboratory of Electronics. Technical Report 248, October 1952.

${ }^{5}$ Greschik, G. et al. "Sensitivity Study of Precision Pressurized Membrane Reflector Deformations." AIAA Journal, Volume 39, No. 2, February 2001.

${ }^{6}$ Coleman, Michael J. and Frank E. Baginski. "Modeling the Shape of Deployable Large Aperture Antennas in Non-symmetric Loading Environments." AIAA Student Region MA-I Conference. April 2009.

7 Coleman, Michael J. and Frank E. Baginski. "A Shape Deformation Study of Large Aperture Inflatable Elastic Parabolic Antenna Reflectors." Eleventh AIAA Gosammer Systems Forum. Orlando, Florida. AIAA 2010 - 2501 (2010). 
${ }^{8}$ Coleman, Michael J. Surface Accuracy Analysis and Mathematical Modeling of Deployable Large Aperture Elastic Antenna Reflectors. Dissertation George Washington University, 2010. Ann Arbor, Michigan: ProQuest UMI, 2010. 108138709.

${ }^{9}$ Baginski, Frank E., Michael C. Barg and William Collier. "Existence Theorems For Tendon-Reinforced Thin Wrinkled Membranes Subjected to a Hydrostatic Pressure Load." Mathematics and Mechanics of Solids 13: 532 - 570, 2008.

10 Baginski, Frank E. and Willi W. Schur. "Structural Analysis of Pneumatic Envelopes: Variational Formulation and Optimization-Based Solution Process." AIAA Journal, Volume 41, No. 2, February 2003.

11 Pipkin, Allen C. "Relaxed Energy Densities for Large Deformations of Membranes." IMA Journal of Applied Mathematics, No. 52, Pages $297-308,1994$.

12 Stutzman, Warren L. and Gary A. Thiele. Antenna Theory and Design. John Wiley and Sons: New York, 1981.

13 Halliday, David and Robert Resnick. Physics Part II. New York: John Wiley and Sons, 1960. 


\begin{tabular}{|c|c|c|}
\hline \multicolumn{2}{|c|}{ REPORT DOCUMENTATION PAGE } & $\begin{array}{l}\text { Form Approved } \\
\text { OMB No. 0704-0188 }\end{array}$ \\
\hline \multicolumn{3}{|c|}{ 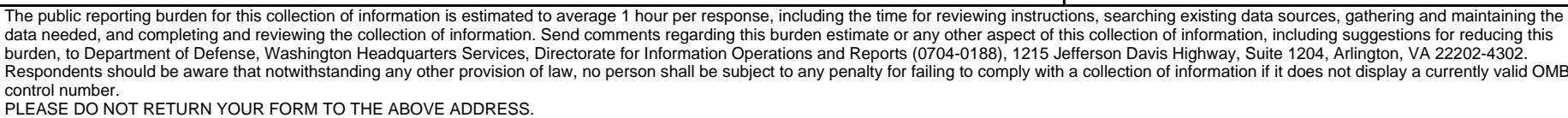 } \\
\hline $\begin{array}{l}\text { 1. REPORT DATE (DD-MM-YYYY) } \\
01-11-2011\end{array}$ & $\begin{array}{l}\text { 2. REPORT TYPE } \\
\text { Technical Memorandum }\end{array}$ & 3. DATES COVERED (From - To) \\
\hline \multirow{3}{*}{\multicolumn{2}{|c|}{$\begin{array}{l}\text { 4. TITLE AND SUBTITLE } \\
\text { The Effect of Boundary Support and Reflector Dimensions on Infla } \\
\text { Performance }\end{array}$}} & 5a. CONTRACT NUMBER \\
\hline & & $\begin{array}{l}\text { 5b. GRANT NUMBER } \\
\text { NNX07AR67G; NNX09AH08G }\end{array}$ \\
\hline & & 5c. PROGRAM ELEMENT NUMBER \\
\hline \multirow{3}{*}{\multicolumn{2}{|c|}{$\begin{array}{l}\text { 6. AUTHOR(S) } \\
\text { Coleman, Michael, J.; Baginski, Frank; Romanofsky, Robert, R. }\end{array}$}} & 5d. PROJECT NUMBER \\
\hline & & 5e. TASK NUMBER \\
\hline & & $\begin{array}{l}\text { 5f. WORK UNIT NUMBER } \\
\text { WBS 439432.04.04.01 }\end{array}$ \\
\hline \multicolumn{2}{|c|}{$\begin{array}{l}\text { 7. PERFORMING ORGANIZATION NAME(S) AND ADDRESS(ES) } \\
\text { National Aeronautics and Space Administration } \\
\text { John H. Glenn Research Center at Lewis Field } \\
\text { Cleveland, Ohio 44135-3191 }\end{array}$} & $\begin{array}{l}\text { 8. PERFORMING ORGANIZATION } \\
\text { REPORT NUMBER } \\
\text { E-17786 }\end{array}$ \\
\hline \multirow{2}{*}{\multicolumn{2}{|c|}{$\begin{array}{l}\text { 9. SPONSORING/MONITORING AGENCY NAME(S) AND ADDRESS(ES) } \\
\text { National Aeronautics and Space Administration } \\
\text { Washington, DC 20546-0001 }\end{array}$}} & $\begin{array}{l}\text { 10. SPONSORING/MONITOR'S } \\
\text { ACRONYM(S) } \\
\text { NASA }\end{array}$ \\
\hline & & $\begin{array}{l}\text { 11. SPONSORING/MONITORING } \\
\text { REPORT NUMBER } \\
\text { NASA/TM-2011-217110 }\end{array}$ \\
\hline
\end{tabular}

\section{DISTRIBUTIONIAVAILABILITY STATEMENT}

Unclassified-Unlimited

Subject Category: 32

Available electronically at http://www.sti.nasa.gov

This publication is available from the NASA Center for AeroSpace Information, 443-757-5802

\section{SUPPLEMENTARY NOTES}

Submitted to Journal of Spacecraft and Rockets

\section{ABSTRACT}

For parabolic antennas with sufficient surface accuracy, more power can be radiated with a larger aperture size. This paper explores the performance of antennas of various size and reflector depth. The particular focus is on a large inflatable elastic antenna reflector that is supported about its perimeter by a set of elastic tendons and is subjected to a constant hydrostatic pressure. The surface accuracy of the antenna is measured by an RMS calculation, while the reflector phase error component of the efficiency is determined by computing the power density at boresight. In the analysis, the calculation of antenna efficiency is not based on the Ruze Equation. Hence, no assumption regarding the distribution of the reflector surface distortions is presumed. The reflector surface is modeled as an isotropic elastic membrane using a linear stress-strain constitutive relation. Three types of antenna reflector construction are considered: one molded to an ideal parabolic form and two different flat panel design patterns. The flat panel surfaces are constructed by seaming together panels in a manner that the desired parabolic shape is approximately attained after pressurization. Numerical solutions of the model problem are calculated under a variety of conditions in order to estimate the accuracy and efficiency of these antenna systems. In the case of the flat panel constructions, several different cutting patterns are analyzed in order to determine an optimal cutting strategy.

\section{SUBJECT TERMS}

Microwave antennas

\begin{tabular}{|c|c|c|c|c|}
\hline \multicolumn{3}{|c|}{ 16. SECURITY CLASSIFICATION OF: } & \multirow{2}{*}{$\begin{array}{l}\text { 17. LIMITATION OF } \\
\text { ABSTRACT } \\
\text { UU }\end{array}$} & \multirow{2}{*}{$\begin{array}{l}\text { 18. NUMBER } \\
\text { OF } \\
\text { PAGES } \\
30\end{array}$} \\
\hline $\begin{array}{l}\text { a. REPORT } \\
U\end{array}$ & $\begin{array}{l}\text { b. ABSTRACT } \\
U\end{array}$ & $\begin{array}{l}\text { c. THIS } \\
\text { PAGE } \\
\text { U }\end{array}$ & & \\
\hline
\end{tabular}

19a. NAME OF RESPONSIBLE PERSON STI Help Desk (email:help@sti.nasa.gov) 19b. TELEPHONE NUMBER (include area code) 443-757-5802 

